\title{
Posterior Shoulder Instability in the Patients with Bilateral Congenital Absence of Long Head of Biceps Tendon: A Case Report
}

\author{
Sung-Hyun Yoon, Kang Heo, Jae-Sung Yoo, Sung-Joon Kim, Joong-Bae Seo \\ Department of Orthopedic Surgery, Dankook University College of Medicine, Cheonan, Korea
}

\begin{abstract}
Rare cases of a congenital absence of the long head of the biceps tendon (LHBT) have been reported, and its incidence is unknown. In a literature review of the congenital absence of the LHBT, only 1 case was associated with posterior shoulder instability and severe posterior glenoid dysplasia. This paper reports the first case of a patient with a bilateral congenital absence of the LHBT with posterior shoulder instability without glenoid dysplasia or posterior glenoid tilt. The patient experienced a traffic accident while holding the gear stick with his right hand. After the accident, a posteroinferior labral tear with paralabral cysts was detected on the magnetic resonance images. The congenital absence of the LHBT was assumed to have affected the posterior instability that possibly increased the susceptibility to a subsequent traumatic posterior inferior labral tear. This case was identified as a posterior inferior tear caused by a traumatic 'gear stick injury'.
\end{abstract} (Clin Shoulder Ellbow 2018;21(4):240-245)

Key Words: Shoulder; Instability; Biceps

Rare cases of a congenital absence of the long head of the biceps tendon (LHBT) have been reported, and its incidence is unknown. In a literature review of congenital absences of the LHBT, 15 cases have been reported, in which 8 cases were bilateral, and 1 case was associated with posterior shoulder instability. ${ }^{1-3)}$ On the other hand, the relationship between an absent LHBT and posterior shoulder instability is difficult to prove through the 1 case associated with posterior shoulder instability because he had severe posterior glenoid dysplasia.

This paper reports a patient with a bilateral congenital absence of the LHBT with posterior shoulder instability. This patient did not have glenoid dysplasia or a posterior glenoid tilt and the absence of the LHBT is believed to have played a role in the unidirectional posterior instability.

\section{Case Report}

\section{History}

A 35-year-old right-handed man, who was also a weight lifter and was employed by an automobile parts supplier, presented with complaints of right shoulder pain. The patient had a traffic accident two months prior. He was driving and holding the gear stick with his right hand (Fig. 1A), and a rear-end collision occurred. The pain did not improve after two months of physical therapy and anti-inflammatory medication. He had no prior history of dislocation or congenital abnormalities.

\section{Physical Exam and Imaging}

The pain worsened during activity, particularly in the endrange of abduction, overhead forward elevation, external rotation, and internal rotation. Both the posterior jerk test and Kim test revealed positive results. Tenderness on the posterior spinoglenoid notch was noted. The patient did not show any signs of anterior instability or multidirectional instability including sulcus signs. There was no generalized laxity. His strength of abduction, external rotation, and internal rotation were all grade 5 . The range of motion of both shoulders was symmetric; there was no 'Popeye' sign to indicate a torn retracted biceps in the distal arm.

Received June 6, 2018. Revised July 25, 2018. Accepted July 30, 2018.

Correspondence to: Joong-Bae Seo

Department of Orthopedic Surgery, Dankook University College of Medicine, 119 Dandae-ro, Dongnam-gu, Cheonan 31116, Korea Tel: +82-41-550-3060, Fax: +82-41-556-3238, E-mail: ssjb1990@dkuh.co.kr, ORCID: https://orcid.org/0000-0003-0334-1049

IRB approval: Dankook University Hospital (No. DKUH 2018-05-012).

Financial support: None. Conflict of interests: None. 

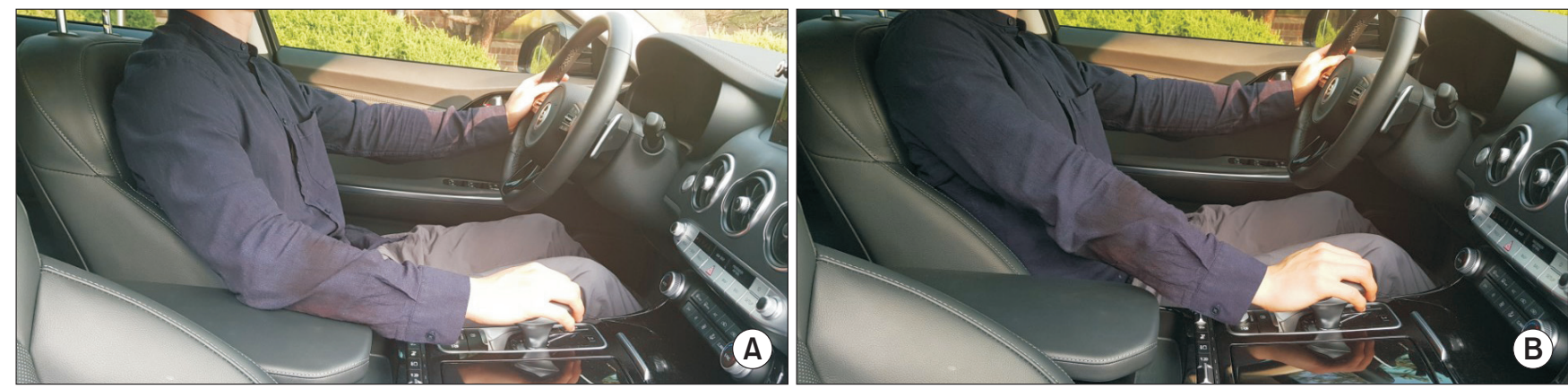

Fig. 1. (A) Lateral view photograph of the driver catching the gear stick. (B) Photograph that is pushing the gear stick in a reflexive manner when a traffic accident occurs.
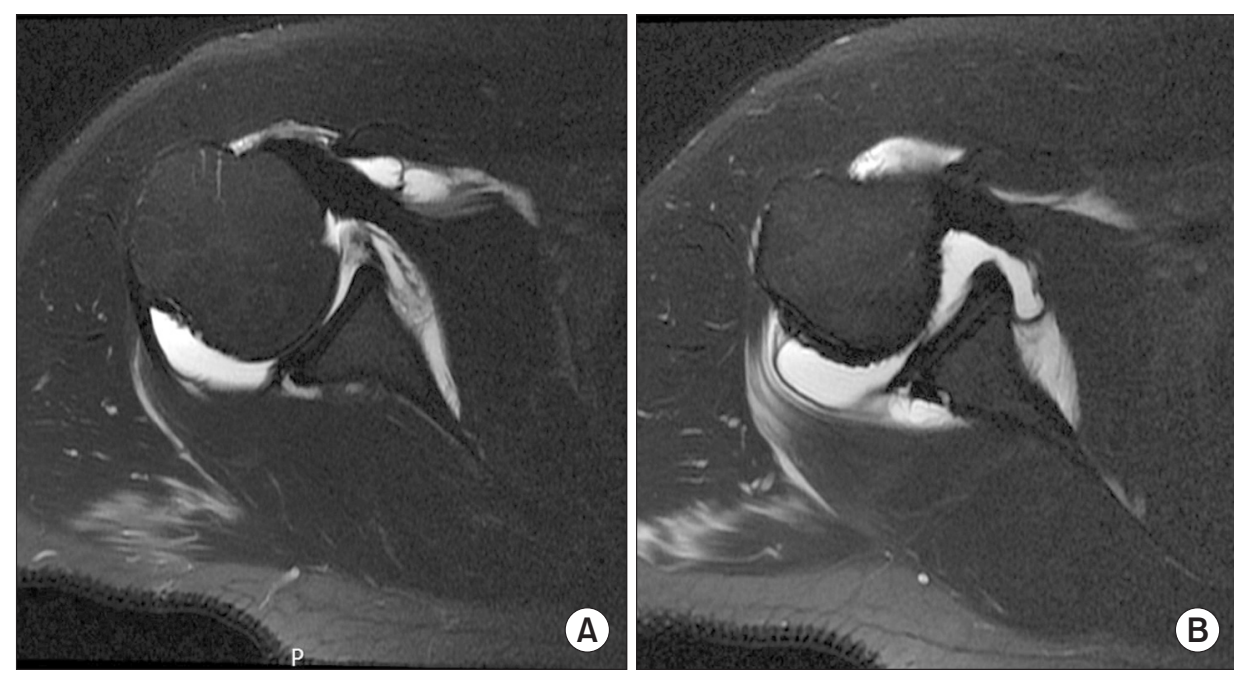

Fig. 2. (A) Magnetic resonance arthrography demonstrated an absence of the long head of the biceps tendon and the shallow, smooth bicipital groove. (B) Magnetic resonance arthrography demonstrated a posteroinferior labral tear with a paralabral cyst.
In addition, the $\mathrm{O}^{\prime}$ Brien test and speed test revealed negative results. He reported no pain when palpating over the course of the LHBT and acromioclavicular or sternoclavicular joints.

Magnetic resonance arthrography (MRA) demonstrated an absence of the LHBT, and there was a shallow, smooth bicipital groove (Fig. 2A). A posteroinferior labral tear with paralabral cysts was observed (Fig. 2B). The continuity of the rotator cuff, including the subscapularis, was preserved. Glenoid dysplasia was not observed $\left(3.5^{\circ}\right.$ of the glenoid retroversion was measured on MRA.

Ultrasonography was performed on both shoulders under the suspicion of a congenital absence of the LHBT; no LHBTs and bicipital grooves were revealed as shallow and smooth appearances in both shoulders (Fig. 3). In the left shoulder, there was no pain, but the posterior jerk test and Kim test revealed positive results with a clunking noise. The patient presented the same sensation of a sudden clunk in those tests, which existed in the right shoulder before the accident. The left shoulder MRI also showed an absence of the LHBT and a shallow bicipital groove, even though a posteroinferior labral tear or paralabral cyst was not observed.

\section{Arthroscopic Findings and Labral Repair}

The patient underwent arthroscopy based on the clinical findings of persistent pain and MRA interpretation. The LHBT was absent on both the posterior and anterior portal. Complete absence of the foramen for the LHBT was also noted (Fig. 4A). No stump of the proximal biceps to suggest a torn retracted biceps was identified (Fig. 4B). In addition, there was no evidence to suggest LHBT adherence to the articular side rotator cuff or joint capsule.

Panlabral wear was noted, and a labral tear was observed on the posteroinferior aspect of the labrum along with paralabral cysts (Fig. 5A). The posteroinferior labrum was repaired with a Juggerknot (Biomet, Warsaw, IN, USA) (Fig. 5B).

\section{Postoperative Rehabilitation}

The patient wore a shoulder immobilizer (Ultrasling ER; Donjoy, Vista, CA, USA) that kept the shoulder at $30^{\circ}$ of external rotation for 6 weeks postoperatively. Only pendulum exercises and scapular retraction were accepted during that period with the shoulder immobilizer. For the next 6 weeks after the immobilization period, the patient was allowed to progressively increase 

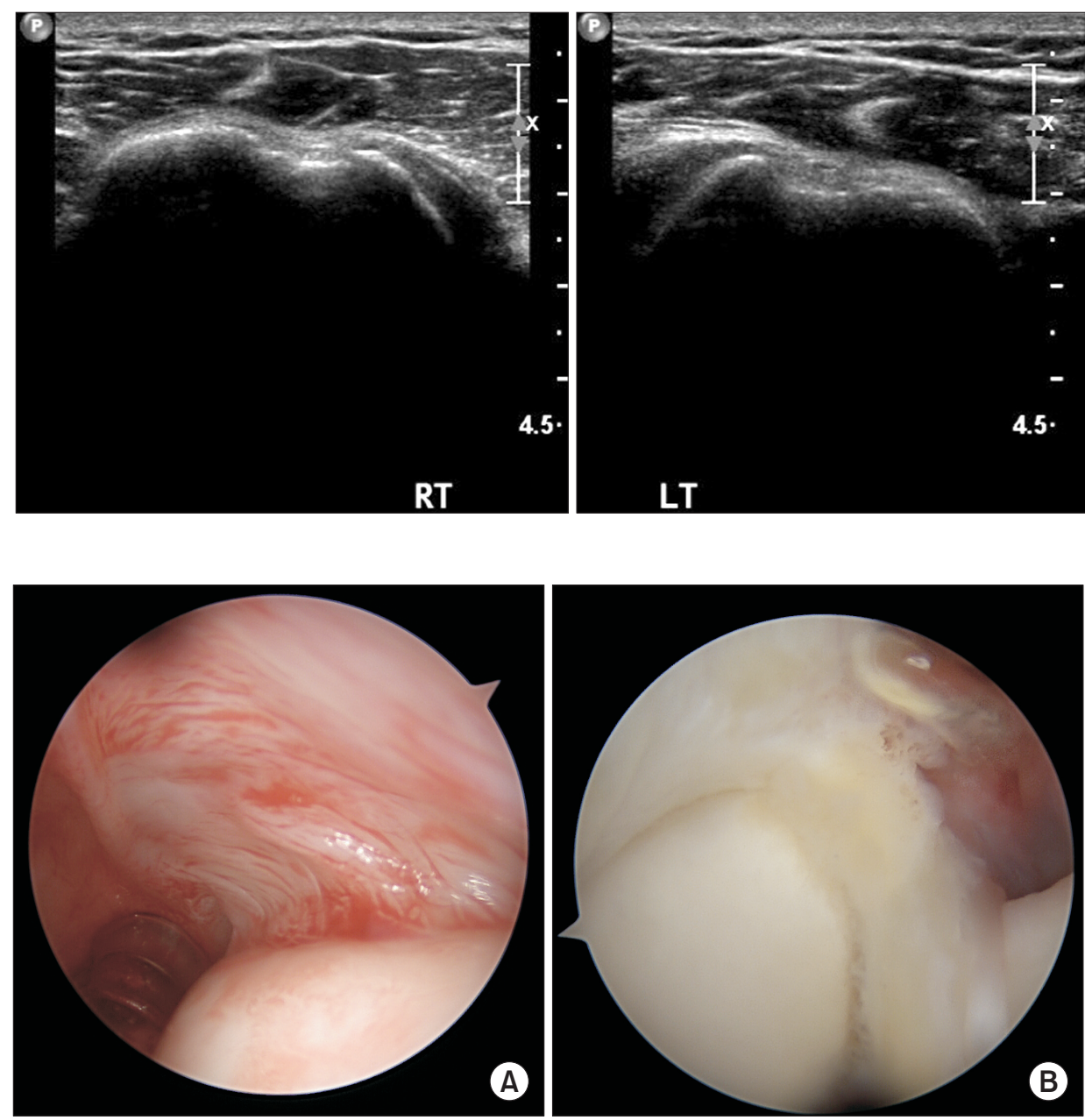

Fig. 4. (A) Arthroscopic view of the rotator interval with the shoulder in the beach chair position. Complete absence of the foramen for the long head of the biceps tendon (LHBT) is noted. (B) Arthroscopic view of the labrum with the shoulder in the beach chair position. Complete absence of the LHBT is noted.
Fig. 3. Both ultrasonography shows no long head of the biceps tendon and shallow, smooth bicipital groove.

RT: right, LT: left.
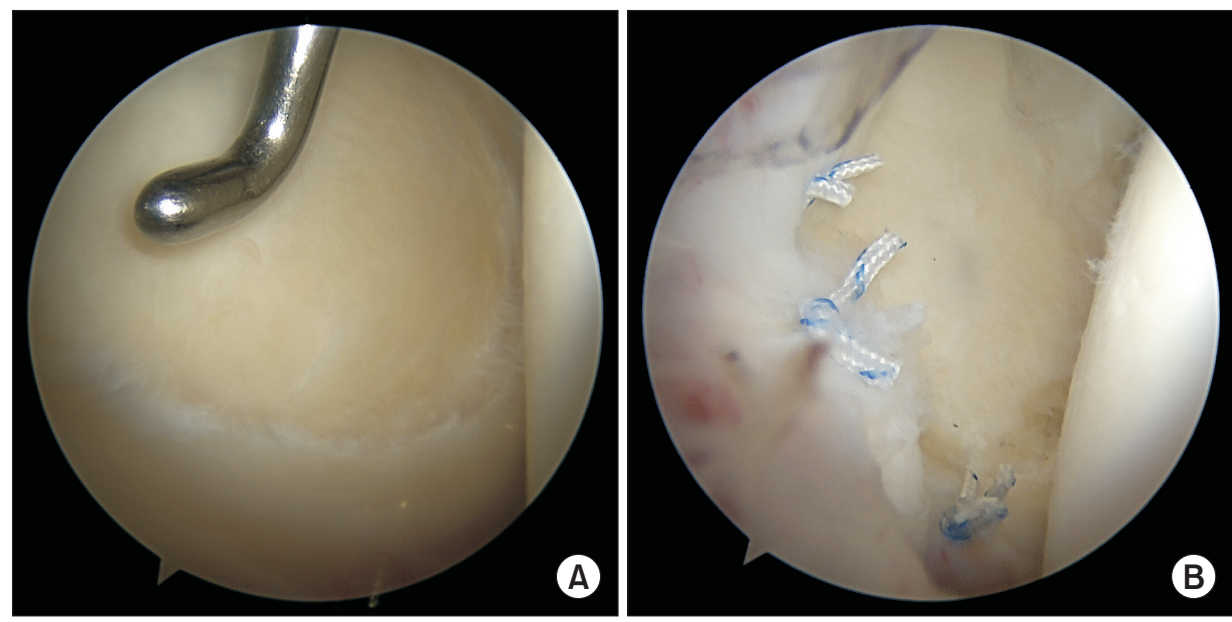

Fig. 5. (A) Arthroscopic view of the inferior aspect of the glenoid with the shoulder in the beach chair position. Posteroinferior (5-9 o'clock) labral tear is noted. (B) Arthroscopic view of the posteroinferior aspect of the glenoid. The labral was repaired with a suture juggerknot $1.5 \mathrm{~mm}$ (Biomet, Warsaw, IN, USA). the range of motion and pain free strength exercises. At 12 to 16 weeks, a more intensive strengthening exercise regimen was allowed and the patient was able to return to work.

\section{Clinical Outcomes}

The patient showed remarkable progress through postopera- tive recovery and rehabilitation. He recovered well for 6 months after discharge and returned to daily life and work without pain. In particular, he did not complain of pain or chronic instability in his work when raising his hands above his head. The pain that had previously occurred on the right side when he was washing his hair or sleeping had also resolved. 


\section{Discussion}

This paper presents a case of bilateral congenital absence of the LHBT in a healthy, 35-year-old man with a posteroinferior labral tear. A congenital anomaly of the LHBT, including complete absence is extremely rare. Kumar et al. ${ }^{1)}$ published a review article that included 13 cases of a complete absence of the LHBT with only 8 cases of a bilateral absence reported until 2017. In South Korea, a case of bilateral absence of the LHBT with anterior instability occurred in 1998, and was not included in the review article. Winston et al. ${ }^{3)}$ reported an additional case of congenital absence of the LHBT in 2017. Among these 15 cases, only one case had signs and symptoms of unidirectional posterior instability. ${ }^{1-3)}$

As observed in other case reports, the patient had a shallow or hypoplastic bicipital groove, with no 'Popeye' deformity in either arm. According to the arthroscopic exam, there were no remnants of the LHBT in both the intra- and extra-articular space. This was considered to be a case of a congenital absence of the LHBT without any other anomaly.

The role of the LHBT has traditionally been described as a humeral head depressor as well as a humeral head compressor. In addition, the LHBT has been reported to be an anterior stabilizer of the shoulder and, more recently, has been found to be a posterior stabilizer as well. ${ }^{4)} \mathrm{A}$ few cases of anterior shoulder instability with an absent LHBT have been reportd." On the other hand, only one case of an absent LHBT associated with posterior labral tear was reported in 2008. In that case, glenoid dysplasia was present with a glenoid retroversion angle of $20^{\circ}$. $^{2)}$ Glenoid dysplasia is one of the known risk factors for recurrent posterior instability. ${ }^{5)}$

In contrast, this patient had a normal range of glenoid retroversion angles $\left(3.5^{\circ}\right)$ and a bilateral congenital absence of the LHBT with unidirectional posterior shoulder instability. Whether the absent LHBT played a role in the patient's posterior inferior labral tear is unclear. On the other hand, considering the patient in this case has no glenoid dysplasia, the authors suggest that the main factor is probably hyperlaxity and the absence of a biceps long head may aggravate his symptoms.

A common traumatic instability observed to occur after a traffic accident is the posterior dislocation of the hip joint. This is commonly called a 'dashboard injury' or 'brake pedal injury'. ${ }^{6,7)}$
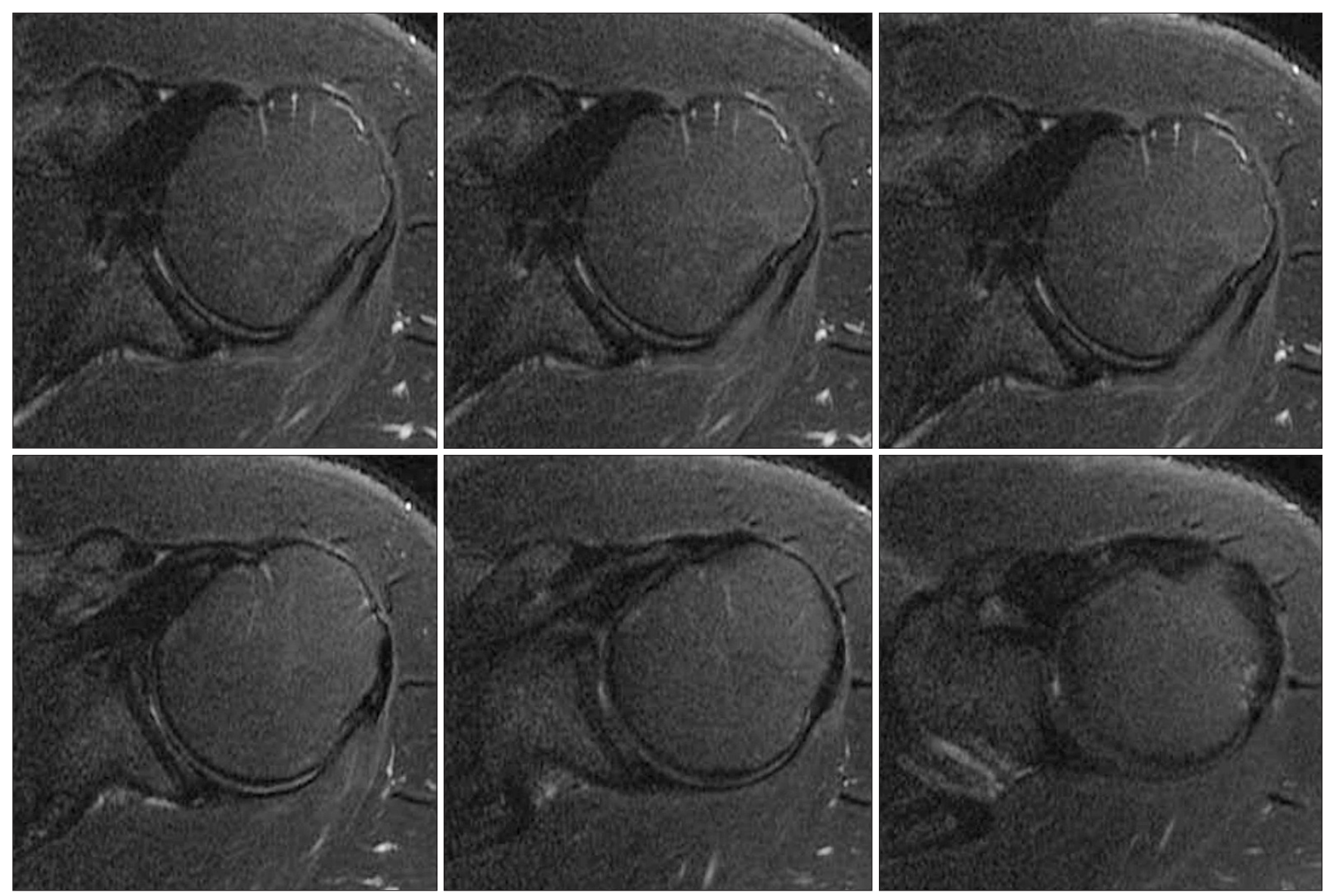

Fig. 6. T2 weighted axial magnetic resonance imagings of the left shoulder. Intraarticular lesion including a labral tear was not detected in uninjured shoulder, even though the left shoulder also had a shallow, smooth bicipital groove with an absence of the long head of the biceps tendon. 

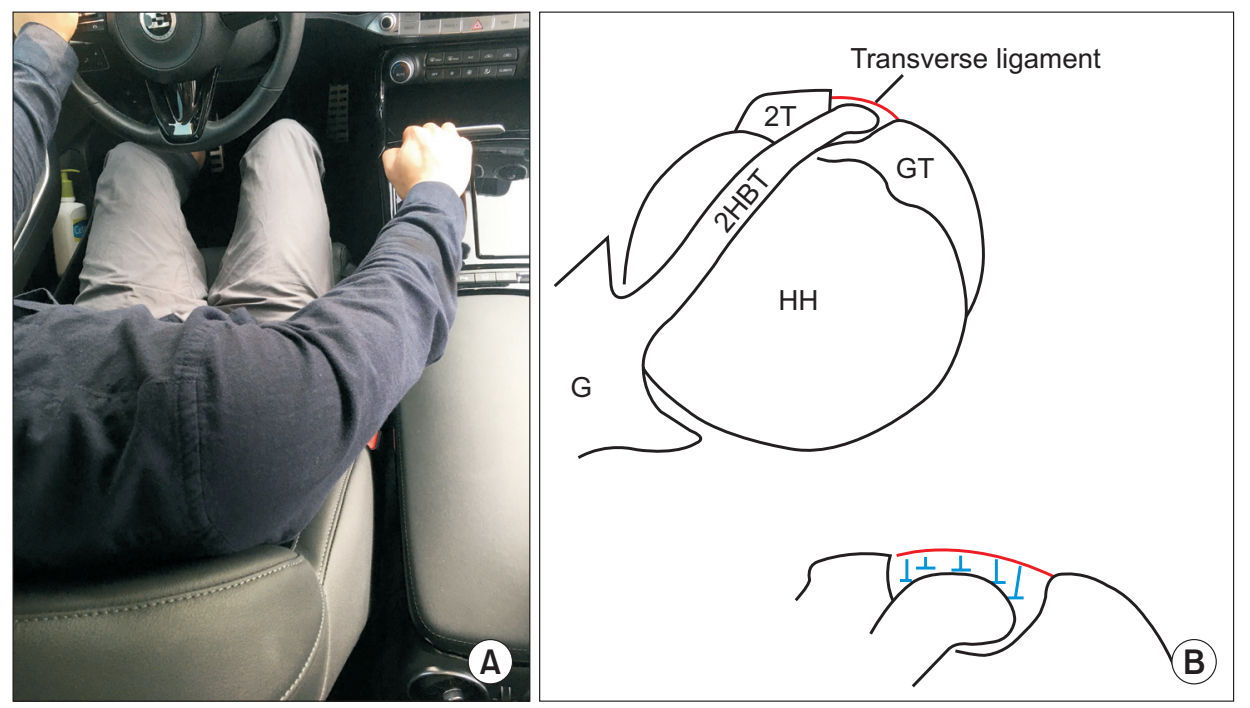

Fig. 7. (A) Photographs of the shoulder position taken from the above when the driver pushed the gear stick reflexively in a traffic accident. (B) The drawing depicts the axial view of the transverse ligament, LHBT, humeral head at the level of bicipital groove. The transverse ligament located in front of the LHBT prevents LHBT from being dislocated in the future by pressing the LHBT, thereby preventing the posterior dislocation of the humeral head.

G: glenoid, HH: humeral head, LHBT: long head of the biceps tendon, GT: greater tuberosity, LT: lesser tuberosity.

The hypothesis of a brake pedal injury is that in rear-end collision accidents, the position of the driver's hip is flexed slightly, adducted, and internally rotated. The driver's knee extends rapidly to press the brake pedal. Subsequently, the load is transferred to the hip joint through the femur, resulting in a series of posterior hip dislocations. ${ }^{6}$ Inferior dislocation in the shoulder has two types of mechanisms, which are classified as direct and indirect injuries. Indirect injuries occur mainly in elderly patients due to the excessive abduction of the shoulder or an external force added to the already abducted shoulder joint. ${ }^{8)}$ Direct injuries are relatively rare. When an external force is applied to an abducted shoulder through the humerus, the humeral head is dislocated inferiorly.

In this case, he presented with the same sensation of a sudden clunk in the jerk and Kim's tests, even though he had no discomfort in the left (uninjured) shoulder. The presence of posterior instability in the right (injured) shoulder before the accident suggest that the posterior instability occurred before the accident and can be classified as atraumatic. On the other hand, he had no posterior labral lesion in the left shoulder, even though an absence LHBT and a shallow, smooth bicipital groove were also observed (Fig. 6).

Moreover, his main complaint was right shoulder pain, which started after the traffic accident. The traffic accident was a rearend collision and the patient's right hand was holding the gear stick. This was defined as a "gear stick injury", and the posterior inferior labral tear and paralabral cyst can be interpreted as traumatic. In addition, the transverse ligament in front of the LHBT may have had a protective role in posterior translation of the humeral head (Fig. 7).

Devgan et al..$^{10)}$ reported a case of a high school wrestler who presented with a rare combination of voluntary posterior shoulder instability combined with superimposed traumatic posterior shoulder instability. The author suggested a combination of a ge- netic predisposition and athletic microtrauma that rendered the inferior glenohumeral ligament loose, increasing his susceptibility to a subsequent traumatic posterior inferior labral tear or reverse Bankart lesion. ${ }^{10)}$ Similarly, the congenital absence of the LHBT may have affected the posterior instability, which might have increased the susceptibility to a subsequent traumatic posterior inferior labral tear.

\section{References}

1. Kumar CD, Rakesh J, Tungish B, Singh DM. Congenital absence of the long head of biceps tendon \& its clinical implications: a systematic review of the literature. Muscles Ligaments Tendons J. 2018;7(3):562-9.

2. Sayeed SA, Shah JP, Collins MS, Dahm DL. Absence of the long head of the biceps tendon associated with glenoid dysplasia and posterior labral tear. Clin Anat. 2008;21(7):728-32.

3. Winston BA, Robinson K, Crawford D. "Monocept": a brief report of congenital absence of the long head of the biceps tendon and literature review. Case Rep Orthop. 2017;2017:1090245.

4. Youm T, ElAttrache NS, Tibone JE, McGarry MH, Lee TQ. The effect of the long head of the biceps on glenohumeral kinematics. J Shoulder Elbow Surg. 2009;18(1):122-9.

5. Brelin A, Dickens JF. Posterior shoulder instability. Sports Med Arthrosc Rev. 2017;25(3):136-43.

6. Monma H, Sugita T. Is the mechanism of traumatic posterior dislocation of the hip a brake pedal injury rather than a dashboard injury? Injury. 2001;32(3):221-2.

7. Funsten RV, Kinser P, Frankel CJ. Dashboard dislocation of the hip: a report of twenty cases of traumatic dislocation. J Bone Joint Surg Am. 1938;20(1):124-32.

8. Karaoglu S, Guney A, Ozturk M, Kekec Z. Bilateral luxatio erecta humeri. Arch Orthop Trauma Surg. 2003;123(6):308- 
10.

9. Mallon WJ, Bassett FH 3rd, Goldner RD. Luxatio erecta: the inferior glenohumeral dislocation. J Orthop Trauma. 1990; 4(1):19-24.
10. Devgan LL, Gill HS, Faustin C, Park HB, McFarland EG. Posterior dislocation in a voluntary subluxator: a case report. Med Sci Sports Exerc. 2006;38(4):613-7. 